\title{
Hasse's primciple for systems of ternary quadratic forms and for one biquadratic form
}

\author{
by
}

A. S C H I N Z E L (Warszawa)

To Professor Jan Mikusinski on the occasion of the 70th birthday

\begin{abstract}
Let $K$ be an algebraic number field and $f_{1}, \ldots, f_{k}$ ternary quadratic forms over $K$. If $f_{1}, \ldots, f_{k}$ have a common non-trivial zero in every completion of $K$ except at most one then - it is proved here - they havie a common non-trivial zero in $K$. Besides an example is given of an absolutely irreducible $n$-ary biquadratic form $(n>3)$ that represents 0 in every completion of $Q$ but not in $Q$.
\end{abstract}

Let $K$ be an algebraic number field and $f_{1}, \ldots, f_{k} \in K\left[x_{1}, \ldots, x_{n}\right]$ quadratic forms. Hasse's principle asserts that if the forms $f_{1}, \ldots, f_{k}$ have a common non-trivial zero in every completion of $\dot{K}$ they have a common non-trivial zero in $K$. The principle holds for $k=1$, it trivially holds for $n=1,2$, and it fails for $K=Q, k=2, n \geqslant 4$ (see [2]). Thus it remains to consider the case $n=3$.

We shall prove

THEOREM 1. If quadratic forms $f_{1}, \ldots, f_{k} \in K[x, y, z]$ have a common non-trival zero in every completion of $K$ except at most one then they have a common non-trivial zero in $K$.

As to biquadratic forms over $Q$ it is easy to give an example of a reducible ternary form for which Hasse's principle fails (see [1], p. 72). An example of an irreducible ternary biquadratic form with the same property can be constructed by using results of Hilbert [4], namely

$$
\operatorname{norm}(x+y \sqrt{5}+z \sqrt{-31}) \text {. }
$$

This form, however, is reducible in the complex field. Mordell [6] has left open the question whether there exists an absolutely irreducible ternary biquadratic form not fulfilling Hasse's principle. The question is answered by 
THEOREM 2. The absolutely irreducible biquadratic form $x^{4}-2 y^{4}-$ $-16 y^{2} z^{2}-49 z^{4}$ represents 0 in every completion of $Q$ but not in $Q$; for alt $n \geqslant 4$ the absolutely irreducible biquadratio form $x_{1}^{4}-17 x_{2}^{4}-2\left(x_{3}^{2}+\ldots+x_{n}^{2}\right)^{2}$ represents 0 in every completion of $Q$ but not in $Q$.

LEMDMA 1. If a binary form over $\mathbb{K}$ of degree not exceeding 4 represents 0 in all but finitely many completions of $K$ it represents 0 in $K$.

Proof. See Fujiwara [3].

LEIMMA 2. Let $R\left(x, y ; u_{1}, \ldots, u_{\gamma_{c}}, v_{1}, \ldots, v_{k_{k}}\right)$ be the resultant of $\sum_{i=1}^{k} u_{i} f_{i}$, $\sum_{i=1}^{k} v_{i} f_{i}$ with respect to $z\left(u_{i}, v_{i}\right.$ are indeterminates $)$. If

(1) $\quad R\left(a, b ; u_{1}, \ldots, u_{k}, v_{1}, \ldots, v_{k}\right)=0, \quad a, b \in K,\langle a, b\rangle \neq\langle 0,0\rangle$

then either $f_{i}$ have a common non-trivial zero in $K$ or

$$
(b x-a y)^{2} \mid R\left(x, y ; u_{1}, \ldots, u_{k}, v_{1}, \ldots, v_{k}\right)
$$

and the forms $f_{i}(a t, b t, z)$ differ from their highest common divisor by a constant factor.

Pro of. If $f_{i}$ are all of degree less than 2 with respect to $z$ then they have a common non-trivial zero, namely $\langle 0,0,1\rangle$. If at least one of the forms $f_{i}$ is of degree 2 with respect to $z$ then both $\sum_{i=1}^{k} u_{i} f_{i}$ and $\sum_{i=1}^{l_{i}} v_{i} f_{i}$ are of degree 2 with respect to $z$ with the leading coefficients independent of $x, y$. Therefore (1) implies that

$$
\sum_{i=1}^{k} u_{i} f_{i}(a, b, z) \quad \text { and } \quad \sum_{i=1}^{k} v_{i} f_{i}(a, b, z)
$$

have a common factor over the field $K\left(u_{1}, \ldots, u_{k}, v_{1}, \ldots, v_{k}\right)$, hence also over the ring $K\left[u_{1}, \ldots, u_{k}, v_{1}, \ldots, v_{k}\right]$. This factor must be independent of $u_{1}, \ldots, u_{k}, v_{1}, \ldots, v_{k^{*}}$. If it is of degree 1 in $z$ it has a zero $c \in K$ and we have $f_{i}(a, b, c)=0(1 \leqslant i \leqslant k)$. If it is of degree 2 in $z$ we consider the Sylvester matrix $S\left(x, y ; u_{1}, \ldots, u_{k}, v_{1}, \ldots, v_{k}\right)$ of the polynomials $\sum_{i=1}^{l_{t}} u_{i} f_{i}$ $\sum_{i=1}^{k} v_{i} f_{i}$. In virtue of a well-known theorem (see [7], Satz 114) the rank of the matrix $S\left(a, b ; u_{1}, \ldots, u_{k}, v_{1}, \ldots, v_{k}\right)$ must be 2 . Hence all the minors of degree 3 of this matrix vanish and all the minors of degree 3 of the matrix $S\left(x, y ; u_{1}, \ldots, u_{k}, v_{1}, \ldots, v_{k}\right)$ are divisible by $b x-a y$. On the other hand, there are minors of degree 2 of the latter matrix not divisible by $b x-a y$, in fact independent of $x, y$. Hence by a very special case of theorem of Rédei [8]

$$
R\left(x, y ; u_{1}, \ldots, u_{k}, v_{1}, \ldots, v_{k}\right)=\operatorname{det} S\left(x, y ; u_{1}, \ldots, u_{k}, v_{1}, \ldots, v_{k}\right)
$$

is divisible by $(b x-a y)^{2}$.

The last assertion of the lemma follows from the remark that if polynomials $f_{i}(a, b, z)(1 \leqslant i \leqslant k)$ have a common factor of degree 2 they differ from this common factor by a constant factor.

Pro of of Theorem 1. Let us consider the resultant $R\left(x, y, u_{1}, \ldots, u_{k}\right.$, $v_{1}, \ldots, v_{k}$ ) of $\sum u_{i} f_{i}$ and $\sum v_{i} f_{i}$ with respect to $z$. Viewed as a polynomial in $x, y$ it is either 0 or a quartic form. In the first case $f_{i}(1 \leqslant i \leqslant k)$ have a common factor, say $d$. If $d$ is of degree 2 then for each $i \leqslant k$ we have $f_{i}=c_{i} d, c_{i} \in K$. The solvability of $f_{i}(x, y, z)=0(i \leqslant k)$ in a completion $K_{v}$ of $K$ implies the solvability of $d(x, y, z)=0$ in $K_{v}$, and if it holds for all but one completion then by the product formula and Hasse's principle for one quadratic form we get solvability in $K$ of $d(x, y, z)=0$ and hence of $f_{i}(x, y, z)=0(1 \leqslant i \leqslant k)$. If $d$ is of degree 1 then it has again a nontrivial zero in $K$ and the same conclusion holds.

If $R\left(x, y ; u_{1}, \ldots, u_{k}, v_{1}, \ldots, v_{k}\right)$ is not identically 0 , let $r(x, y)$ be the highest common divisor of its coefficients when viewed as a form in $u_{1}, \ldots, u_{k}, v_{1}, \ldots, v_{k}$. If $f_{i}(x, y, z)(1 \leqslant i \leqslant k)$ have a common non-trivial zero $\left\langle a_{v}, b_{v}, c_{v}\right\rangle$ in $K_{v}, \sum u_{i} f_{i}$ and $\sum v_{i} f_{i}$ have it also, hence $R\left(a_{v}, b_{v}\right.$; $\left.u_{1}, \ldots, u_{k}, v_{1}, \ldots, v_{k}\right)=0$, which implies

$$
r\left(a_{v}, b_{v}\right)=0 .
$$

(Here we use the fact that the coefficients of $R$ are forms in $x, y$ ). If $a_{v}=b$ $=0$ we have $c_{v} \neq 0$; hence the coefficient of $z^{2}$ in $f_{i}$ is 0 for each $i \leqslant k$ and the forms $f_{i}(1 \leqslant i \leqslant k)$ have in $K$ a common non-trivial zero $\langle 0,0,1\rangle$. If $\left\langle a_{v}, b_{v}\right\rangle \neq\langle 0,0\rangle$ for each valuation $v$ of $K$ except at most one then by Lemma $1 r$ has in $K$ a zero, say $\langle a, b\rangle \neq\langle 0,0\rangle$. Thus $b x-a y \mid r(x, y)$,

$$
b x-a y \mid R\left(x, y ; u_{1}, \ldots, u_{k}, v_{1}, \ldots, v_{k}\right)
$$

and by Lemma 2 either $f_{i}$ have a common non-trivial zero in $K$ or

$$
(b x-a y)^{2} \mid R\left(x, y ; u_{1}, \ldots, u_{k}, v_{1}, \ldots, v_{k}\right)
$$

and the forms $f_{i}(a t, b t, z)(1 \leqslant i \leqslant k)$ differ from their highest common divisor by a constant factor. In the latter case, by (3)

$$
(b x-a y)^{2} \mid r(x, y) \text {. }
$$


Let

$$
r(x, y)=(b x-a y)^{\alpha} s(x, y)
$$

where $a \geqslant 2, s(a, b) \neq 0, \operatorname{deg} s=\operatorname{deg} r-\alpha \leqslant 2$. For every valuation $v$ of $K$ except at most one we have by (2) and (4)

$$
b a_{v}-a b_{v}=0 \quad \text { or } \quad s\left(a_{v}, b_{v}\right)=0 .
$$

The first equation implies $a_{v}=a t, b_{v}=b t$ for a $t \in K_{v}^{*}$; thus

$$
F(t, u)=s(t, u) \underset{1 \leqslant i \leqslant k}{\text { h.c.d. }} f_{i}(a t, b t, u)
$$

has a non-trivial zero in $K_{v}$. Since by (4)

$$
\operatorname{deg} F=\operatorname{deg} s+2=\operatorname{deg} r+2-\alpha \leqslant 4,
$$

we infer from Lemma 1 that $F$ has in $K$ a zero, say $\langle c, d\rangle \neq\langle 0,0\rangle$. If this is a zero of the $\underset{1 \leqslant i \leqslant k}{\text { h.c.d. }} f_{i}(a t, b t, u)$, then

$$
f_{i}(a c, b c, d)=0 \quad(1 \leqslant i \leqslant k), \quad\langle a c, b c, d\rangle \neq\langle 0,0,0\rangle .
$$

If, on the other hand, $s(c, d)=0$ then by $(4) r(c, d)=0$; thus

$$
R\left(c, d ; u_{1}, \ldots, u_{k}, v_{1}, \ldots, v_{k}\right)=0
$$

and by Lemma 2 either $f_{i}$ have a common non-trivial zero in $K$ or

$$
(d x-c y)^{2} \mid R\left(x, y ; u_{1}, \ldots, u_{k}, v_{1}, \ldots, v_{k}\right)
$$

and $f_{i}(c t, d t, z)(1 \leqslant i \leqslant k)$ differ by a constant factor from their highest common divisor. In the latter carse

$$
(d x-c y)^{2} \mid r(x, y)
$$

and by (4)

$$
r(x, y)=e(b x-a y)^{2}(d x-c y)^{2} \text {. }
$$

For every valuation $v$ of $K$ except at most one we have by (2)

$$
b a_{v}-a b_{v}=0 \quad \text { or } \quad d a_{v}-c a_{v}=0 ;
$$

thus for a suitable $t \in K_{v}^{*}$ either $a_{v}=a t, b_{v}=b t$ or $a_{v}=c t, b_{v}=d t$. It follows that the quartic form

$$
G(t, u)=\underset{1 \leqslant i \leqslant k}{\text { h.c.d. }} f_{i}(a t, b t, u) \cdot \underset{1 \leqslant i \leqslant k}{\text { h.c.d. }} f_{i}(c t, d t, u)
$$

has a non-trivial zero in $K_{v}$. By Lemma $1 G(t, u)$ has in $K$ a zero, say $\left\langle t_{0}, u_{0}\right\rangle$ $\neq\langle 0,0\rangle$. If $\left\langle t_{0}, u_{0}\right\rangle$ is a zero of the h.c.d. $f_{i}(a t, b t, u)$ then

$$
f_{i}\left(a t_{0}, b t_{0}, u_{0}\right)=0 \quad(1 \leqslant i \leqslant k), \quad\left\langle a t_{0}, b t_{0}, u_{0}\right\rangle \neq\langle 0,0,0\rangle ;
$$

if $\left\langle t_{0}, u_{0}\right\rangle$ is a zero of the $\underset{1 \leqslant i \leqslant k}{\text { h.c.d. }} f_{i}(c t, d t, u)$ then

$$
f_{i}\left(c t, d t_{0}, u_{0}\right)=0 \quad(1 \leqslant i \leqslant k), \quad\left\langle c t_{0}, d t_{0}, u_{0}\right\rangle \neq\langle 0,0,0\rangle .
$$

The proof is complete.

For the proof of Theorem 2 we need three lemmata.

LEMMra 3. The equation $u^{4}-17 v^{4}=2 w^{2}$ has no solutions in $Q$ except $\langle 0,0,0\rangle$.

Proof. See Lind [5] or Reichardt [10].

LEMMCA 4. Let $F\left(x_{1}, \ldots, x_{n}\right)$ be a polynomial with integer $p$-adic coefficients and $\gamma_{1}, \ldots, \gamma_{n} p$-adic integers. If for an $i \leqslant n$ we have

$$
\begin{gathered}
F\left(\gamma_{1}, \ldots, \gamma_{n}\right) \equiv 0\left(\bmod p^{2 \delta+1}\right), \\
\frac{\partial F}{\partial x_{i}}\left(\gamma_{1}, \ldots, \gamma_{n}\right) \equiv 0\left(\bmod p^{\delta}\right), \\
\frac{\partial F}{\partial x_{i}}\left(\gamma_{1}, \ldots, \gamma_{n}\right) \neq 0\left(\bmod p^{\delta+1}\right)
\end{gathered}
$$

( $\delta$ a nonnegative integer) then there exist $p$-adic integers $\theta_{1}, \ldots, \theta_{n}$ such that

$$
F\left(\theta_{1}, \ldots, \theta_{n}\right)=0
$$

and $\theta_{1} \equiv \gamma_{1}\left(\bmod p^{\delta+1}\right), \ldots, \theta_{n} \equiv \gamma_{n}\left(\bmod p^{\delta+1}\right)$.

Proof. See [1], p. 42 .

LeMca 5. $f(x, y, z)=x^{4}-2 y^{4}-16 y^{2} z^{2}-49 z^{4}$, is irreducible in every field of characteristic different from 2 and 17.

Proof. Let $k$ be a field of this kind. It is enough to show that $f(x, y, z)$ is irreducible as a polynomial in $x$ over $k(y, z)$. If it were not, then by Capelli's theorem (see [9], Satz 428) $\pm\left(2 y^{4}+16 y^{2} z^{2}+49 z^{4}\right.$ ) would have to be a square in $k(y, z)$. This condition implies that

$$
16^{2}-4 \cdot 2 \cdot 49=-8 \cdot 17=0 \text {, }
$$

which is possible only if $\operatorname{char} k=2$ or $\operatorname{char} k=17$.

Proof of Theorem 2. $f(x, y, z)=x^{4}-17 z^{4}-2\left(y^{2}+4 z^{2}\right)^{2}$; hence by Lemma 3 if $f(x, y, z)=0$ and $x, y, z \in Q$ we have $x=y^{2}+4 z^{2}=0$ and thus $x=y=z=0$. Also $x_{1}^{4}-17 x_{2}^{4}-2\left(x_{3}^{2}+\ldots+x_{n}^{2}\right)^{2}=0$ implies $x_{1}=x_{2}=\ldots=x_{n}=0$ for $x_{i} \in Q$. 
It remains to show that $f(x, y, z)$ represents 0 in every field $Q_{p}$ including $Q_{\infty}=R$. We verify this first using Lemma 3 for $p=\infty, 2,5,7,13$ and 17 .

For $p=\infty$ we take $x=\sqrt[4]{2}, y=1, z=0$.

For $p=2$ we use Lemma 4 with $\gamma_{1}=3, \gamma_{2}=2, \gamma_{2}=1, \delta=2, i=1$.

For $p=5$ we use Lemma 4 with $\gamma_{1}=0, \gamma_{2}=2, \gamma_{3}=1, \delta=0, i=2$.

For $p=7$ we use Lemma 4 with $\gamma_{1}=2, \gamma_{2}=1, \gamma_{3}=0, \delta=0, i=1$.

For $p=13$ we use Lemma 4 with $\gamma_{1}=1, \gamma_{2}=2, \gamma_{3}=3, \delta=0$, $i=1$.

For $p=17$ we use Lemma 4 with $\gamma_{1}=0, \gamma_{2}=1, \gamma_{3}=2, \delta=0$, $i=2$.

For $p \neq 2,5,7,13,17$ we have either $p \geqslant 37$ or for a suitable $\operatorname{sign}( \pm 7 \mid p)=1$

In the latter case the congruence

$$
f(x, 0, z)=\left(x^{2}-7 z^{2}\right)\left(x^{2}+7 z^{2}\right) \equiv 0(\bmod p)
$$

is solvable nontrivially, and denoting its solution by $\gamma_{1}, \gamma_{3}$ we use Lemma 4 . with $\gamma_{2}=0, \delta=0, i=1$.

It remains to consider primes $p \geqslant 37$. For such primes $f$ is by Lemma 5 absolutely irreducible over $F_{p}$. Moreover, it has no singular zeros. Indeed, the equations

$$
4 x^{3}=0, \quad-8 y^{3}-32 y z^{2}=0, \quad-32 y^{2} z-196 z^{3}=0
$$

imply $x=0$ and either $y=0,196 z^{3}=0$ or $y^{2}+4 z^{2}=0,68 z^{2}=0$; thus in any case $x=y=z=0$. By the Riemann-Hurwitz formula the curve $f(x, y, z)=0$ is over $F_{p}$ of genus 3 .

Therefore by Weil's theorem the number of points on this curve with coordinates in $F_{p}$ is greater than $p+1-6 \sqrt{p}$, i.e., at least one. Since all points are non-singular, Lemma 4 applies with $\delta=0$ and a suitable $i$.

Note added in proof. I have learned that already in 1981 A. Bremner, D. J. Lewis and P. Morton found the example $3 x^{4}+4 y^{4}-19 z^{4}$ of a ternary biquadratic form for which Hasse's principle fails, but they did not publish it.

\section{References}

[1] Z. I. Borevich and I. R. Shafarevich, Number Theory, New York and London 1966.

[2] J.-L. Colliot-Thélène, D. Coray, J.-J. Sansuc, Descente et principe de Hasse pour certaines variétés rationnelles, J. Reine Angew. Math. 320 (1980), 150-191.

[3] M. Fujiwara, Hasse principle in algebraic equations, Acta Arith. 22 (1973) 267-276. [4] D. Hilbert, Über Diophantische Gleichungen, Nachr. Königl. Gesell. Wiss.
Göttingen 1897, 48-54.
[5] C. E. Lind, Untersuchungen über die rationalen Punkte der ebenen kubischen Kurven vom Geschlecht Eins (Doctoral dissertation), Uppsala 1940.

[6] L. J. Mordell, The diophantine equation $A x^{4}+B y^{4}+C z^{4}=0$, Proc. Cambridge Philos. Soc. 68 (1970), 125-128.

[7] 0. Perron, Algebra I, Berlin 1951.

[8] L. Rédei, Ủber die Determinantenteiler, Acta Math. Acad. Sci. Hungar. 3 (1952), $143-149$.

[9] - Algebra I, Leipzig 1959.

[10] H. Reichardt, Einige im Kleinen überall lösbare, im Grossen unlösbare diophantische Gleichungen, J. Reine Angew. Math. 184 (1942), 12-18.

Received June 2, 1982 